\title{
Kernos
}

Revue internationale et pluridisciplinaire de religion grecque antique

12| 1999

Varia

\section{KRINGS, Carthage et les Grecs c. 580-480 av. J.-C.}

\section{Corinne Bonnet}

\section{(2) OpenEdition}

\section{Journals}

Édition électronique

URL : http://journals.openedition.org/kernos/751

DOI : 10.4000/kernos.751

ISSN : 2034-7871

Éditeur

Centre international d'étude de la religion grecque antique

Édition imprimée

Date de publication : 1 janvier 1999

Pagination : 327-331

ISSN : 0776-3824

Référence électronique

Corinne Bonnet, « V. KRINGs, Carthage et les Grecs c. 580-480 av. J.-C. », Kernos [En ligne], 12 | 1999, mis en ligne le 13 avril 2011, consulté le 10 mars 2021. URL : http://journals.openedition.org/kernos/751 ; DOI : https://doi.org/10.4000/kernos.751 
démographie, 343 au sujet de l'époque hellénistique) pourrait laisser sur sa faim le public encore peu aguerri auquel il s'adresse, une lacune que ne comble pas le choix éditorial de ne pas présenter de documents (quelques citations de textes anciens dans les deux premières parties toutefois). Les 18 illustrations (on signalera un léger décalage entre la pagination réelle et les pages reprises dans la table) sont constituées par des cartes originales sauf celle de la colonisation grecque archaïque, p. 112-113, reprise à H. Bengtson. En outre, aux p. 477-485, sont proposés d'utiles tableaux généalogiques (les Antigonides, les Ptolémées, les Séleucides, les Attalides et les souverains du Pont). Vu l'abondance des questions traitées et le volume de ce manuel universitaire, et malgré une table des matières analytique très détaillée, on regrettera l'absence d'un index.

Véronique Krings

Véronique Krings, Carthage et les Grecs c. $580-480$ av. J.-C. Textes et bistoire, Leiden, Brill, 1998. 1 vol. $16 \times 24,5 \mathrm{~cm}$, XIII +427 p. (Studies in the History and Culture of the Ancient Near East, 13). ISBN : 90-04-10881-5.

L'ouvrage de Véronique Krings, issu d'une thèse de doctorat présentée à l'Université de Liège en 1996, est avant tout un livre d'historienne qui s'interroge sur les mécanismes de reconstitution du passé et sur les éventuelles interférences que le présent peut occasionner dans ce processus. À cet égard, on peut dire que la portée de ce livre «transcende » largement le cas spécifique des relations entre Carthage et les Grecs, et l'on saura gré à l'A. d'avoir mis sa propre réflexion "épistémologique » au service d'un domaine qui se cherche encore sur le plan méthodologique - le domaine des études phénico-puniques - et qui, pour diverses raisons «structurelles», a trop longtemps été dominé par des sortes de «vulgates » scientifiques, émanant d'autorités « historiques» en la matière, dont il est sans doute temps de commencer à s'affranchir, sans pour autant renier l'apport «fondateur» de leurs écrits. On se plaira à souligner que, sur ce plan, l'ouvrage de Véronique Krings fait preuve de grande pondération dans l'évaluation du parcours historiographique qu'elle sonde et retrace. À lire son essai, on est en droit de se demander s'il est vraiment fortuit que des lectures aussi novatrices que celle qui est ici proposée se fasse jour dans le domaine phénicopunique un an seulement après la disparition de Sabatino Moscati. Les grands maîtres des études phénico-puniques, dont S. Moscati est assurément le dernier en date, ont, pourrait-on dire, à la fois consacré des modèles interprétatifs (du reste en partie hérités de leurs propres prédécesseurs) et contribué dans une large mesure, par leur ouverture d'esprit, à forger les instruments qui, dans les mains d'une nouvelle génération, plus sensible à d'autres thématiques et à d'autres méthodes, permettent de remettre en question ces modèles et de les dépasser. L'histoire a besoin, nul n'en doutera, de «schémas » interprétatifs, de «clés de lecture » qui, en extrapolant du particulier au général, génèrent le sens de l'histoire, lui confèrent une unité, dessinent les lignes d'un développement, dégagent les ressorts de la causalité. Il s'agit là d'un exercice à la fois indispensable et risqué. Pour l'histoire de Carthage (et du monde phénicien), une toute récente «ère des synthèses », pour reprendre l'heureuse expression d'un des derniers articles de S. Moscati (L'età delle sintesi, dans Rivista di studi fenici 23, 1995 , p. 127-146), a conclu une longue période de recherches intenses; les monographies, et autres dictionnaire ou manuel, se sont multipliés, qui ont permis de faire le point sur nos connaissances et qui ont souvent consacré une certaine vision de l'évolution historique de Carthage et du monde phénico-punique en général. Mais dans quelle mesure ces «vulgates » sont-elles encore suffisamment nourries de la lecture (ou relecture) et de l'examen direct, attentif, critique, approfondi, 
des sources - la sève de l'historien - et non pas plutôt d'une sorte de consensus historiographique ? Véronique Krings a choisi de procéder à une nouvelle autopsie - terme cher à Thucydide - des rapports entre Carthage et les Grecs, d'en disséquer tous les nouds, d'en mettre à vif tous les ressorts, bref de démonter la machine historiographique. Propos iconoclaste en somme, mais constructif aussi, nous allons le voir, qui ne pouvait venir - mon expérience de l'historiographie du $\mathrm{xx}^{\mathrm{e}}$ siècle sur les traces de Franz Cumont m'en a convaincue - que d'une «marginale» (sans aucune connotation péjorative, évidemment), une Belge, de formation classique, sans attache académique, loin de tout conformisme, avec un bel esprit critique, mûr et exigeant, sans arrogance ni polémique. Et l'on pourrait noter, dans la même ligne, que ce livre met bien en évidence l'apport décisif de l'historiographie espagnole récente à ce renouvellement de perspectives, ce qui confirme une certaine "décentralisation " du savoir et son affranchissement progressif des modèles-vulgates (cf. notamment le volume de Eduardo Ferrer Albelda, España cartaginesa. Claves bistoriograficas para la bistoria de España, Sevilla, 1996).

La Liste Royale Sumérienne, avec ses diverses dynasties mésopotamiennes, probablement rédigée au $2^{\mathrm{e}}$ millénaire, est sans doute le premier document historique qui témoigne de la volonté d'organiser le passé en une succession de "dynasties» ou d' « empires » (au sens étymologique du terme). Horror vacui d'une part qui suggère aux rédacteurs de remplir les blancs pour que la chaîne de l'histoire - l'ADN de l'humanité en somme - ne connaisse aucune fracture, volonté de lire le passé d'autre part comme la manifestation d'une volonté divine qui fait et défait les royaumes. La même conception «théologique » et téléologique de l'histoire sous-tend la célèbre prophétie de Daniel relative à la succession des empires qui, dans un univers désormais gagné à l'« historicisme » inhérent au christianisme, s'impose comme un modèle historiographique majeur. Les Carolingiens avec l'idée de renovatio imperii en assureront la transmission, et après eux le «Saint Empire Germanique », pour ne pas parler des Bourguignons (et de leurs célèbres réminiscences antiques) et des manifestations plus récentes encore, comme les empires napoléonien ou prussien. Le fait est que la logique des empires, donc des blocs qui se font concurrence, se combattent et se supplantent, est omniprésente dans l'historiographie moderne. Yalta et son implaca ble logique - symbolisée par le défunt Mur de Berlin - imposa définitivement, et pendant des décennies, le modèle d'un monde un peu schizofrène, où seuls existent des rapports de force, des blocs, des antagonistes, la guerre froide. Du reste dans l'Antiquité, de telles oppositions avaient déjà été savamment soulignées : Athènes-Sparte, Grecs-Perses, Romains-Carthaginois : une Méditerranée qui préfigure étrangement l'Europe divisée de l'après-guerre, ce qui n'a pas manqué de frapper les historiens et d'influencer leur interprétation des événe ments antiques auxquels ils ont appliqué une grille de lecture moderne. Le dualisme et le manichéisme (bons/méchants; civilisés/barbares; conquérants/ indigènes) y trouvèrent largement leur compte, mais l'histoire ?

L'intrusion des sciences sociales dans l'histoire a beaucoup fait pour renouveler l'approche de ces questions. La Méditerranée archaïque a-t-elle vraiment été le théâtre d'affrontements de type impérialiste ? N'aurions-nous pas aussi, à bien y regarder, les traces tangibles d'une autre logique, d'une autre dynamique, faite de rencontres et de contacts ( incontri », dirait-on en italien, et non «scontri ») ? L'anthropologie historique a heureusement mis l'accent sur l'importance et la complexité des mécanismes d'inter-relation culturelle entre les peuples et sur les subtiles dynamiques d'acculturation différenciée qui accompagnent les phénomènes d'expansion commerciale et/ou politique depuis l'Antiquité. 
D'autre part - et c'est un autre aspect essentiel du travail de Véronique Krings - la philologie moderne ne se satisfait plus d'une Quellenforscbung linéaire et « génétique » qui se limiterait à indiquer pour chaque source la ou les « parents » du témoignage. Il s'agit désormais de reconstituer «l'itinéraire de l'information» qui, tel un cours d'eau, charrie progressivement des éléments neufs, accueille des affluents, sans cesse mélange ses eaux et n'est en somme jamais la même en aucun point de son parcours; un témoignage n'est donc jamais parfaitement « reconductible » à un autre, même s'il en dérive. Enfin, plus que jamais, on est attentif à deux dimensions du témoignage écrit : celle de l'idéologie qui l'imprègne, le traverse et le modèle; celle de la composition avec ses exigences et ses techniques qui peuvent créer de sérieuses distorsions au sein de la matière historique. Car, durant toute l'Antiquité, l'histoire est bien - et là réside une bonne partie de l'ambiguîté de notre démarche et de notre terminologie - un genre littéraire, soumis à des impératifs de composition (au demeurant complexes, $c f$. p. 333) et de «marché » (tendances moralisatrices, patriotisme ou chauvinisme, vulgarisation), et non pas une pratique scientifique, professionnelle, avec sa «déontologie » propre, comme celle qu'ont ou devraient avoir les historiens d'aujourd'hui.

On trouvera sur ces questions des considérations très stimulantes dans les conclusions de Véronique Krings, aux pages 327-369. Leur richesse explique pourquoi le volume ne s'ouvre que sur un bref avant-propos pour nous plonger immédiatement dans le vif du sujet. En somme, l'A. met en œuvre sa méthode et en tire les enseignements en fin de volume, afin de permettre au lecteur de la juger «sur pièces ». Six chapitres constituent le corps de l'enquête, six épisodes significatifs des rapports entre Carthage et les Grecs dans l'intervalle qui va de 580 environ à $480 \mathrm{av}$. J.-C., un siècle d'histoire de la Méditerranée 'occidentale, à la charnière entre la période archaïque et classique. Il s'agit de «L'affaire Pentathlos », «L'énigme "Malchus" », «Les leçons d'Alalia », «Les infortunes de Dorieus », «L'"inconnue" Artémision » et enfin «Himère : au Couchant comme au Levant ». La question (multiforme) qui sous-tend l'enquête pourrait être formulée en ces termes : est-il légitime de parler d' «impérialisme » carthaginois pour cette période ? est-il légitime de lire les six épisodes traités, qui sont les seuls points d'appui de la reconstruction historique, comme la pointe d'un iceberg, celui du prétendu conflit entre Carthage et les Grecs pour le contrôle économique, politique et culturel de la Méditerranée occidentale ? est-il légitime de relier entre eux ces pointillés et d'en proposer une lecture « unificatrice »?

Nous laisserons au lecteur le soin de prendre directement connaissance des six dossiers et d'évaluer la lecture et l'interprétation des sources que propose l'A. La grande majorité de ses propositions devraient, me semble-t-il, susciter sans difficulté l'adhésion des spécialistes. On relèvera que la structure des six chapitres est semblable et traduit fort bien la démarche de l'A. Il s'agit d'en revenir aux textes anciens, de partir d'eux, sans à priori (autant que faire se peut) et en enquêtant au maximum sur le contexte spécifique de chaque témoignage ( «contextualiser " est le néologisme fréquemment employé par l'A.) : la chronologie, l'orientation et la structure de l'œuvre, sa transmission et sa réception, la formation de l'auteur, sa vision de l'histoire, ses modèles, sa conception du temps et de l'espace, les interférences idéologiques, les procédés de composition littéraire, les sources de l'information (concept d' «itinéraire » de l'information), le ou les publics visés, les questions terminologiques (par exemple la mention de Phéniciens, de Puniques ou de Carthaginois). Ainsi voit-on Hérodote, Diodore, Pausanias, Orose ou Justin, pour ne citer que l'un ou l'autre des auteurs pris en considération, passés en quelque sorte à la moulinette. Une fois les témoignages «traqués » sous toutes leurs facettes, on passe alors aux propositions interpré- 
tatives que ceux-ci permettent de formuler, une tentative, en somme, de baliser le terrain de la reconstruction historique de points de repère sûrs, même s'ils peuvent sembler «minimalistes ». Ces acquis sont alors confrontés avec les différentes reconstructions proposées par les prédécesseurs de l'A. : la grande scène de l'histoire apparaît trop souvent comme une fiction et l'envers du décor en révèle toute la fragilité et subjectivité. On soulignera ici le soin apporté à l'établissement des textes ainsi qu'aux traductions, et la richesse des références bibliographiques. Dans le registre des qualités générales du volume, on n'omettra pas de signaler l'excellente présentation formelle et le nombre très réduit de coquilles, y compris dans les langues étrangères abondamment citées en notes pour reporter in extenso les passages historiographiques les plus significatifs.

Mettons à présent en évidence les apports majeurs, les lignes de force du travail de Véronique Krings.

D'abord, la constatation, bien documentée, que l'histoire, dans le sens de l'enregistrement du passé, n'est pas univoque; elle procède de mécanismes cognitifs complexes qui souvent superposent divers niveaux de réalités. Dans le cas qui nous occupe, il est très clair que sur la lecture des rapports de Carthage avec les Grecs par les historiens de l'époque hellénistique et romaine (la majorité de nos témoignages) se projettent deux ombres «déformantes » : celle de l'idéologie césarienne ou augustéenne, qui impose un certain modèle de gestion du pouvoir auquel les événements du passé sont, consciemment ou non, rapportés, d'où des distorsions; celle aussi des Guerres Puniques qui crée une fausse perspective historique en vertu de laquelle tout ce qui précède préfigure la conflictualité chronique entre Carthage et ses interlocuteurs en Méditerranée occidentale. Carthage, en somme, comme archétype de l'« adversaire ».

Ensuite, on retiendra le travail de «décodage» d'une historiographie moderne qui, trop souvent, construit l'identité historique de Carthage archaïque sur l'idée d'imperium héritée des Anciens, relayée par l'historiographie du Reich et " confirmée ", entérinée par les grandes synthèses de ces dernières années. À cet égard, les pages 87-91 sont exemplaires : elles montrent comment la figure de Malchus a été l'objet d'une surévaluation et surinterprétation historiographique, au point de devenir un chaînon indispensable de la reconstruction historique. L'argumentation circulaire aidant, l'interprétation d'un épisode local circonscrit et au demeurant fort incertain devient dogme et acquiert, qui plus est, une valeur générale dans un cadre méditerranéen. Déconstruire, dans ces cas-là, devient une tâche constructive dans la mesure où cela permet un retour aux sources, à leur questionnement critique et à une nouvelle proposition de réécriture (ou de relecture, sur la différence, $c f$. p. 341) de l'histoire.

Enfin, cet ouvrage propose un réajustement de perspective sur ce qui s'est en définitive passé en Méditerranée occidentale entre c. 580 et 480. Plutôt que l'image d'une Méditerranée coupée au couteau, hérissée de barrières défensives et théâtre de conflits chroniques, on doit privilégier le souvenir d'un espace où s'enchevêtrent des courants commerciaux nombreux, des intérêts économiques variés (y compris ceux de la piraterie), des expressions culturelles en interaction constante. D'où une coexistence relativement pacifique, scandée de temps à autre d'incidents, d'accrochages, dont l'ancrage local ou régional, souvent, n'a pas échappé aux sources, mais a été l'objet d'une surenchère de la part des modernes. «Du civilisationniste à l'événementiel», qui est le titre d'un paragraphe des conclusions, invite donc à éviter les généralisations faussement rationnelles (par exemple les discours sur les équilibres "politiques " en Méditerranée) et insuffisamment documentées pour se limiter plus modestement, mais plus solidement à une «histoire en pointillés », c'est-à-dire à l'examen des «événements » 
sur lesquels les sources apportent vraiment des éclairages. Mais ici, il importe de bien comprendre le propos de l'A. qui n'est pas de verser dans le positivisme, c'est-à-dire dans l' «hystérie » de la reconstitution factuelle minutieuse, mais au contraire de rendre à chacun sa "vérité ", c'est-à-dire sa lecture des événements, que l'on ne peut comprendre qu'en la «contextualisant ", c'est-à-dire en la relativisant. Un retour à l'événement donc, mais au sein d'une conception selon laquelle "l'interprétation de l'histoire fait partie intégrante de celle-ci ». Dans une perspective comme celle-là, loin de tout idéalisme absolu, on évite le piège de la hiérarchisation des témoignages pour saisir la valeur, la portée et la signification intrinsèques de chacune de ses productions humaines, donc historiques.

Autant de considérations qui dépassent largement le cas de Carthage et qui devraient encourager tous les historiens de l'Antiquité à lire le beau livre de Véronique Krings.

Corinne Bonnet

(FNDP Namur - Pontificio Istituto Biblico Università di Cosenza)

\section{Actes de colloques, ouvrages collectifs et mélanges}

Antonetri Claudia (éd.), Il dinamismo della colonizzazione greca. Atti della tavola rotonda 'Espansione e colonizzazione greca di età arcaica: metodologie $e$ problemi a confronto' (Venezia, 10-11/11/1995), Napoli, Loffredo, 1997.

G. Mangarano, Mondo religioso greco e mondo 'indigeno' in Sicilia, p. 71-82; C. AntonetTi, Megara e le sue colonie: un'unità storico-culturale?, p. 83-94; P. SIEWERT, Privilegien überseeischer Griechen im Heiligtum von Olympia, p. 95-96.

Bosch M. del C., Fornés M.A. (éds.), Homenatge a Miquel Dolc. Actes del XII Simposi de la Secció Catalana i I de la Secció Balear de la SEEC, Palma, 1 al 4 de febrer de 1996, Palma de Mallorca, 1997.

Alberto BernabÉ, Orfeotelestas, intérpretes, charlatanes: transmisores de la palabra órfica, p. 37-41; Francesc CASADEsús BordoY, Orfics o impostors? El testimoni d'Eurípides: Hipòlit 943-957, p. 167-170; Francesc J. Cuartero Iborra, Demèter a Eleusis (Ps. Apollodor, Biblioteca 15 , 1s. 131 s.J, p. 183-188; Manuel García Teijeiro, El tema de la amante fantasma desde Flegón, p. 194-198

Boulogne Jacques (éd.), Les systèmes mythologiques, Lille, Presses univ. du Septentrion, 1997.

J. Boulogne, Pour une approche systémique de la mythologie grecque. Le cas de Médée, p. 209-234; A. Morgau, Jason et Oreste. D'un système à l'autre : du béros épique au misérable, p. 271-282; P. WATHELET, Le mythe d'Ulysse, p. 283-293.

Bouvier David, Calame Claude (éds), Pbilosophes et bistoriens anciens face aux mythes, publié dans Études de Lettres. Revue de la Faculté des lettres de l'Univ. de Lausanne, 1998, fasc. 2.

Lucio Bertelli, Des généalogies mythiques à la naissance de l'bistoire : le cas d'Hécatée; Monica Visintin, La colère de Minos : à propos d'Hérodote, VII, 169-71, p. 3342; Enzio Pellizer, Le petit-fils de Zeus : la légende de Télèphe entre mythe et histoire, p. 43-56; Silvia Subli Milanezi, Athènes : mythe comique; représentations d'Athènes et des Athéniens dans la comédie d'Aristophane, p. 59-72; Claude Mossá, La construction d'un mythe bistorique : la Vie de Lycurgue de Plutarque, p. 83-87; Michel Fatral, Mythe et 